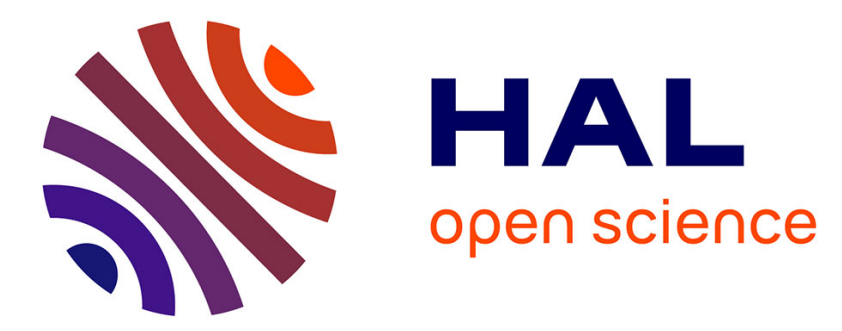

\title{
La formation en sciences pharmaceutiques, des réformes successives
}

\author{
Élisabeth Adenot, Laetitia Agay, Jean-Paul Belon, Arthur Piraux
}

\section{To cite this version:}

Élisabeth Adenot, Laetitia Agay, Jean-Paul Belon, Arthur Piraux. La formation en sciences pharmaceutiques, des réformes successives. Actualités Pharmaceutiques, 2019, 58, pp.20 - 24. 10.1016/j.actpha.2018.12.005 . hal-03486074

\section{HAL Id: hal-03486074 https://hal.science/hal-03486074}

Submitted on 20 Dec 2021

HAL is a multi-disciplinary open access archive for the deposit and dissemination of scientific research documents, whether they are published or not. The documents may come from teaching and research institutions in France or abroad, or from public or private research centers.
L'archive ouverte pluridisciplinaire HAL, est destinée au dépôt et à la diffusion de documents scientifiques de niveau recherche, publiés ou non, émanant des établissements d'enseignement et de recherche français ou étrangers, des laboratoires publics ou privés.

\section{다)(1) $(5$}

Distributed under a Creative Commons Attribution - NonCommercial| 4.0 International 
Version of Record: https://www.sciencedirect.com/science/article/pii/S0515370018304865

Manuscript_af0d02d299d3538478b261ccf5a46952

Dochead dossier

Sous-dochead Les nouveaux enjeux de la formation du pharmacien

\title{
La formation en sciences pharmaceutiques, fruit de réformes successives
}

\author{
Élisabeth Adenot ${ }^{\mathrm{a}, *}$, \\ Étudiante en troisième année des études pharmaceutiques, secrétaire générale et attachée \\ de presse de l'Anepf \\ Laetitia Agay \\ Pharmacienne, vice-présidente en charge de la communication à l'Anepf 2016-2017 \\ Jean-Paul Belon ${ }^{d}$ \\ Professeur des Universités, ancien membre de la CPNEP et de la CPNES

\section{Arthur Piraux ${ }^{\mathrm{e}}$}

Pharmacien, vice-président en charge de l'enseignement supérieur à l'Anepf 2016-2017

aUniversité Paris-Descartes, 4 avenue de l'Observatoire, 75006 Paris, France

${ }^{b}$ Anepf, 4 avenue Ruysdaël, 75008 Paris, France

${ }^{`} 6$ chemin du Hameau-Blanc, 38240 Meylan, France

dUFR des sciences de santé, Université de Bourgogne, 7 boulevard Jeanne-d'Arc, 21000 Dijon, France

e 68 chemin des Bordages, 72230 Arnage, France

*Auteur correspondant.

Adresse e-mail : presse@anepf.org (É. Adenot).

Résumé

En France, les études de santé sont réglementées au niveau national. La formation pharmaceutique a fait l'objet de nombreux décrets et arrêtés fixant les différentes modifications induites par les

(C) 2018 published by Elsevier. This manuscript is made available under the CC BY NC user license https://creativecommons.org/licenses/by-nc/4.0/ 
réformes successives.

(c) 2018

Mots clés - cursus LMD ; diplôme d'État de docteur en pharmacie ; enseignement ; études de pharmacie

Summary à venir

(c) 2018

Keywords à venir

L'arrêté du 19 juin 1980 est un texte fondateur pour la formation en sciences pharmaceutiques. II marque la fin de la réglementation du régime des études par décret [1]. II précise notamment les points concernant les dispositions générales, la formation (enseignements avec contenu et volumes horaires), le contrôle des connaissances, les stages, la thèse, ainsi que les dispositions diverses afférentes à l'abrogation du décret fondamental du 26 novembre 1962 (encadré 1).

\section{Encadré 1. Le décret du 26 novembre 1962}

Le décret du 26 novembre 1962 était le premier à apporter autant de précisions quant au contenu des études et au volume horaire dédié à chaque matière d'enseignement. Il précisait aussi leur durée : cinq années sanctionnées par le diplôme de pharmacien.

Ce décret a été modifié les années suivantes, notamment par la loi d'orientation de l'enseignement supérieur du 12 novembre 1968 (loi Edgar Faure) qui créa les universités : les facultés et écoles de pharmacie devinrent des unités d'enseignement et de recherche (UER) des sciences pharmaceutiques ou des UER mixtes de sciences médicales et pharmaceutiques. Ces UER furent ensuite transformées en unités de formation et de recherche (UFR) par la loi ${ }^{\circ} 84-52 \mathrm{du}$ 26 janvier 1984. Le décret du 8 novembre 1968 portant organisation des études en vue du diplôme de pharmacien précisait les modalités des enseignements dans le cadre défini de trois options : officine, industrie et biologie.

Sur 1 colonne près de son appel

\section{T1 Réglementation des études de pharmacie}

TEG1 Les études pharmaceutiques en vue du diplôme d'État de docteur en pharmacie sont largement réglementées par l'arrêté du 19 juin 1980 qui modifie le décret $n^{\circ} 73-227$ du 27 février 1973 relatif aux diplômes nationaux de l'enseignement supérieur. Il supprime les termes "diplôme d'État de pharmacien" et "certificats d'études supérieures de pharmacie" au profit des dénominations "diplôme d'État de docteur en pharmacie" (le décret n 80-1097 du 
24 décembre 1980 oblige les étudiants en pharmacie à soutenir une thèse pour l'obtention du diplôme [2]) et "diplôme d'études approfondies dans les disciplines pharmaceutiques". II modifie aussi très sensiblement le décret de 1962 en faisant apparaître les stages hospitaliers dont les modalités seront régies par le décret 91-1102 du 15 décembre 1981. Enfin, il précise l'unicité du diplôme ${ }^{1}$.

TEG1 L'arrêté du 12 septembre 1985 est également essentiel car il fait passer la durée des études à six années et dessine ainsi l'aspect général actuel de la formation pharmaceutique [3]. Il instaure un premier et un deuxième cycle de deux années chacun ainsi qu'un troisième cycle comportant une cinquième année hospitalo-universitaire suivie, soit d'une sixième année à orientation professionnelle, soit de quatre années de spécialisation pour les étudiants reçus au concours de l'internat [4], ouvrant à la formation afférente aux diplômes d'études spécialisées (DES). L'arrêté fait aussi apparaître une spécificité à l'échelon européen : l'année hospitalo-universitaire (stage annuel mi-temps en milieu hospitalier organisé en quadrimestres). Il introduit également :

- la notion d'unités de valeur (UV) d'enseignement, qui remplacent les modules d'enseignements ;

- une formation commune de base comprenant des enseignements non optionnels ainsi qu'une formation optionnelle au cours des deux premiers cycles pour permettre à l'étudiant d'approfondir ses connaissances de base dans les disciplines de son choix et le préparer progressivement à un exercice professionnel particulier ou à une carrière de recherche ;

- la discipline de pharmacie clinique.

La sixième année supplémentaire comprend un stage de pratique professionnelle de six mois, des enseignements théoriques dirigés ou pratiques et la soutenance d'une thèse.

TEG1 L'arrêté du 17 juillet 1987 relatif au régime des études en vue du diplôme d'État de docteur en pharmacie [5] reprend et modifie les textes parus depuis le décret de 1962. II crée (art. 35) la Commission pédagogique nationale des études pharmaceutiques (CPNEP) chargée notamment de réviser et d'actualiser les programmes des enseignements de la formation en les réglementant au niveau national. Un enseignement de synthèse est également conçu au premier trimestre de la cinquième année pour permettre à l'étudiant d'intégrer l'ensemble des acquis. Une initiation à la recherche sous la forme d'un stage est proposée de façon facultative à partir de la deuxième année d'études ; cette formation particulière fait référence à la maîtrise des sciences biologiques et médicales (MSBM) qui avait pour but de permettre l'accès aux diplômes d'études approfondies (DEA) en offrant un complément de connaissances aux étudiants des formations de santé. Pour obtenir la MSBM, il fallait valider trois certificats, la validation d'un stage d'initiation à la recherche (150 heures) pouvant remplacer un certificat. Cet arrêté initial de 1987 a été successivement modifié par les arrêtés des 29 octobre 1992, 7 novembre 1994, 29 octobre 1996, 20 mai 1998 et 2 juillet 1999.

\section{T1 Les besoins professionnels pris en compte}

TEG1 Tous ces arrêtés ont été régulièrement partiellement complétés et actualisés afin d'adapter les études aux nouveaux besoins professionnels ou/et de se conformer aux règlements en vigueur. Une actualisation importante devenait à nouveau impérative, notamment à la suite de la parution du 
rapport du Comité national d'évaluation (CNE), créé par la loi n 84-52 du 26 janvier 1984 [6], concernant la formation des pharmaciens en France [7]. En effet, la formation pharmaceutique devait s'accorder impérativement à l'évolution, considérable en quelques années, des métiers de la pharmacie. De plus, elle devait inclure la notion de partage de compétences et de transfert de tâches avec d'autres professionnels de santé.

TEG1 Dans le même esprit, dès l'année 1998, la CPNEP a accompli sa tâche de révision et d'actualisation en profondeur du contenu des enseignements et des modalités pédagogiques afférentes, en s'appuyant notamment sur l'évaluation des 24 UFR de pharmacie réalisée durant la période 1996-1998 par le CNE [8]. Les travaux de la commission se sont concrétisés avec la parution de l'arrêté du 14 août 2003 [9]. Ce dernier relevait l'importance des stages en milieu professionnel et mettait en exergue l'application d'une nouvelle méthode pédagogique, préconisant des enseignements coordonnés dont la mise en œuvre était conseillée dès la deuxième année des études. Cette pédagogie a pour objectif d'aider l'étudiant à développer ses capacités de synthèse et son esprit critique. L'ensemble des UFR l'a globalement adoptée, certes de manière inégale, mais avec des résultats encourageants et globalement positifs. Elle s'applique sans difficulté dans la démarche licence-master-doctorat (LMD) car elle s'adapte à la souplesse attachée aux European Credits Transfer System (ECTS) créés en 1988 par l'Union européenne. Cette méthode pédagogique facilite également la validation de modules d'enseignement dans le cadre des échanges internationaux (Erasmus, Leonardo).

\section{T1 L'adaptation du cursus au schéma LMD}

TEG1 La CPNEP a poursuivi ses travaux en s'attachant en premier lieu à l'actualisation du programme des études pour les mettre en conformité avec la démarche LMD appliquée en France aux formations de santé à la suite du discours du ministre en charge de l'éducation nationale, François Fillon, à la Conférence des présidents d'université (CPU), le 21 octobre 2004. La France n'applique pas le processus de Bologne aux formations conduisant à des professions réglementées dans le domaine de la santé du fait de la nécessité de maintenir un cursus encadré sur le plan national ; elle a cependant adopté le principe de la semestrialisation et des ECTS. Dans ce contexte, il était devenu indispensable de repenser le cadre général de la formation pour adapter le parcours des études pharmaceutiques à cette démarche qui redéfinissait également les cycles d'études à l'échelle européenne. Le décret $n^{\circ}$ 2002-482 du 8 avril 2002 [10] appliqué aux études supérieures et aux diplômes nationaux fixe une architecture des études fondée sur trois grades : licence (bac +3 , 180 crédits), master (bac $+5,300$ crédits) et doctorat (bac $+8,480$ crédits).

TEG1 Les études de pharmacie se prêtent aisément au schéma LMD malgré un cursus particulier de six années d'études dont la première fait l'objet d'un concours particulier. La Commission paritaire nationale de l'emploi (CPNE), dans son rapport final relatif à la pharmacie d'officine de mars 2006 [11], ainsi que l'Ordre national des pharmaciens, dans son livre blanc publié en janvier 2007 [12], ont confirmé l'intérêt de l'adhésion au système LMD.

TEG1 Le socle commun de connaissances (SCC) pharmaceutique renferme des connaissances générales scientifiques et de santé, ainsi que des connaissances spécifiques (unités 
d'enseignement [UE] de pré-orientation ou de pré-spécialisation) suffisantes pour guider et permettre le choix d'une orientation professionnelle raisonnée dès l'acquisition de la licence (soit une année plus tôt que dans le schéma de la formation pharmaceutique précédent).

Ade_illus1

\section{T1 La première année en question}

TEG1 La première année des études pharmaceutiques était sanctionnée par un concours spécifique, l'examen était classant avec un numerus clausus fixé par arrêté interministériel [6]. Elle a fait l'objet d'une réflexion particulière orientée sur la possibilité de mettre en place un concours commun à d'autres professions de santé. Une commission pédagogique nationale de la première année des études de santé, créée en avril 2002 et dirigée par le professeur Domitien Debouzie, a étudié la faisabilité d'une première année de formation et de sélection pour 14 professions de santé. Le rapport final donnait un exemple de programme commun d'enseignement ${ }^{2}$ qui aurait pu être dispensé dans ce cas de figure [13]. La CPNEP a été associée à cette réflexion et a émis un certain nombre de propositions pour l'enseignement afférent aux études spécifiques de pharmacie.

TEG1 La loi $n^{\circ}$ 2009-833 portant création d'une première année commune aux études de santé (Paces) et facilitant la réorientation des étudiants a été promulguée le 7 juillet 2009 [14]. La CPNEP a contribué activement à l'élaboration du programme commun de Paces, notamment en proposant la création d'un module d'enseignement en rapport avec le médicament (UE6 "Initiation à la connaissance du médicament"). Ce programme d'enseignement a été réglementé par l'arrêté du 28 octobre 2009 (version consolidée du 29 août 2017) [15]. La possibilité d'admission (procédure de passerelles) en deuxième ou en troisième année des études médicales, odontologiques, pharmaceutiques ou de sage-femme, de même que la possibilité d'exercer un droit au remords et la fixation du nombre d'étudiants admis à poursuivre les études en fin de Paces ont été réglementés par quatre arrêtés respectifs publiés le 26 juillet 2010.

\section{T1 De nouveaux contenus}

TEG1 Dans le même temps, la CPNEP a engagé des travaux importants de modifications du programme des enseignements postérieurs à ceux de l'année de Paces. L'élaboration d'un référentiel général des métiers de la pharmacie (inexistant jusqu'alors), décliné en trois référentiels spécifiques - "activités", "formation" et "compétences" - était nécessaire car les objectifs pédagogiques devaient s'appuyer sur ces documents pour prendre en compte l'évolution des métiers de la pharmacie et définir avec précision le contenu du schéma LMD appliqué aux études pharmaceutiques : licence (trois années), master (deux années) et troisième cycle court (une année).

TEG1 L'ensemble des contenus (ECTS) des enseignements, ainsi que leur importance, place et articulation adaptées au schéma européen (capitalisation, validation de semestres et d'années) ont été fixés pour chacune des étapes du SCC. Dans l'esprit du LMD, les enseignements de préorientation et de pré-spécialisation ainsi que la progression adaptée de certains d'entre eux ont été 
définis pour permettre à l'étudiant de choisir son parcours d'orientation professionnelle (à l'issue du $\mathrm{SCC}$ ) et préciser également l'adaptation de ce parcours à un cursus plus spécialisé. Pour la rédaction des référentiels, la commission s'est appuyée sur les documents officiels émis par le Conseil de l'Europe et notamment, pour la filière officine, les résolutions AP (94)1 sur l'usage rationnel des médicaments, AP (97)2 sur l'évolution de la fonction du pharmacien et l'adaptation de sa formation initiale, AP (2001)2 sur le rôle du pharmacien dans le cadre de la sécurité sanitaire, ainsi que la Directive 2005/36/CE relative à la reconnaissance des qualifications professionnelles [16]. Pour la rédaction particulière des référentiels afférents aux métiers de l'industrie, la commission a sollicité la collaboration du Leem (Les entreprises du médicament). Pour la problématique générale de rédaction des référentiels, elle a suivi les conseils de la Direction générale de la santé (DGS) et de la Direction de l'hospitalisation et de l'organisation des soins (DHOS) du ministère de la Santé.

\section{TEG1 Ces référentiels métiers concernent les quatre orientations professionnelles (officine,} biologie, industrie, internat) correspondant aux principaux métiers de la pharmacie et qui font l'objet de parcours de formation spécifiques et peuvent comporter certaines particularités selon les UFR. Trois niveaux d'acquisition de connaissances ont été fixés pour chaque référentiel "compétences" afin de préciser et de répartir graduellement les enseignements correspondants à chaque étape de la formation (encadré 2). Le programme d'enseignement et les modalités associées ont été définis à partir de ces documents. Le contenu intégral de la formation s'accorde avec l'unicité du diplôme, car il s'appuie sur les référentiels des différents métiers de la pharmacie.

\section{Encadré 2. Trois niveaux d'acquisition de connaissances}

La Commission pédagogique nationale des études pharmaceutiques (CPNEP) a établi le cadre général de la formation et ses objectifs en précisant dans le détail les niveaux progressifs de connaissances théoriques et pratiques afférentes aux compétences à chaque étape du parcours :

- un premier niveau (diplôme de formation générale en sciences pharmaceutiques [DFGSP]), correspond au socle commun de compétences que l'étudiant doit acquérir quelle que soit I'orientation professionnelle choisie ; ces compétences partagées définissent la première étape du socle commun de connaissances (SCC), lequel concerne la totalité de la licence et le premier semestre de master ;

- un second niveau (diplôme de formation appliquée en sciences pharmaceutiques [DFASP]) équivaut à un enseignement théorique et pratique plus spécifique, adapté au parcours afférent à l'orientation professionnelle, dispensé au cours des trois autres semestres de master ;

- un troisième niveau (diplôme d'État de docteur en pharmacie) se rapporte à la formation en lien direct avec la pratique professionnelle (stage[s] et enseignements complémentaires de spécialisation), qui est dispensée au cours de la sixième année (troisième cycle court). 


\section{T1 L'internat en pharmacie}

TEG1 L'internat en pharmacie a été réglementé par des arrêtés et décrets successifs dès 1973 et notamment par le décret $n^{\circ}$ 89-739 du 12 octobre 1989 modifié par le décret $n^{\circ}$ 2001-37 du 11 janvier 2001 [17]. Le Centre national des concours d'internat (CNCl) a quant à lui été créé par l'arrêté du 23 juin 1998 [18]. Le concours d'accès à l'internat a lieu en décembre de chaque année. Il est possible de concourir deux fois à partir de la quatrième année validée. À l'issue des délibérations, les étudiants, classés en rang utile, choisissent leur filière, leur inter-région et, dans un deuxième temps, leur premier terrain de stage, en fonction de leur classement. Depuis la dernière grande réforme des diplômes d'études spécialisées [19], l'internat ouvre aux trois DES suivants : biologie médicale, pharmacie, innovation pharmaceutique et recherche.

TEG1 Tous les DES comprennent une formation pratique qui s'effectue dans des services agréés (stages semestriels) et une formation théorique assurée par l'UFR et le centre hospitalier universitaire (CHU). Ils sont organisés en huit semestres de formation répartis en deux niveaux :

- le premier permet à chaque étudiant de valider les différents domaines socles de chaque discipline ;

- le second vise une spécialisation selon l'option choisie au sein de chaque DES.

Les DES constituent des formations spécialisantes d'une durée de quatre années. Tout comme l'exercice de la biologie médicale est réservé aux titulaires du DES de biologie médicale, l'exercice en pharmacie à usage intérieur (PUI) l'est aux seuls titulaires du DES de pharmacie depuis la parution du décret du 9 mai 2017 [20].

\section{Points à retenir}

- Le système universitaire actuel se base sur un modèle européen de validation d'European Credits Transfer System (ECTS).

- L'internat ouvre sur trois diplômes d'études spécialisées (DES) : biologie médicale, pharmacie hospitalière, innovation et recherche.

- Depuis 2010, des expérimentations ont lieu sur le territoire pour réformer la première année commune aux études de santé (Paces).

- Les étudiants en pharmacie doivent soutenir une thèse afin d'acquérir le grade de docteur en pharmacie.

- Le système d'évaluation est en pleine réforme : fin de la Paces, DES officine, DES industrie, DES spécialités pharmaceutiques et recherche (SPR), approche par compétences, amendement des stages...

Sur 1 colonne près de son appel 


\section{T1 Les deux arrêtés fondateurs}

TEG1 L'ensemble des travaux de la réforme des études pharmaceutiques réalisés par la CPNEP et poursuivis par l'inter-commission de pharmacie au sein de la Commission de pédagogie nationale des études de santé (CPNES) [21], chargée de formuler des avis et de faire des propositions sur les orientations et le déroulement de ces études, s'est matérialisé par la publication de deux arrêtés. Ces derniers réglementent les études pharmaceutiques au niveau national dans la démarche LMD :

- l'arrêté du 22 mars 2011 relatif au régime des études en vue du diplôme de formation générale en sciences pharmaceutiques (le DFGSP sanctionne la licence pharmaceutique et ouvre l'accès au[x] master[s] pharmaceutique[s]) [22] ;

- l'arrêté du 8 avril 2013 relatif au régime des études en vue du diplôme d'État de docteur en pharmacie (le diplôme de formation approfondie en sciences pharmaceutiques [DFASP] permet I'accès au troisième cycle court qui se termine avec la soutenance de la thèse de doctorat d'état en pharmacie) [23].

TEG1 L'inter-commission pharmacie de la CPNES a complété la publication de ces deux arrêtés par la rédaction du descriptif détaillé des compétences à acquérir au cours des stages en lien avec les objectifs pédagogiques définis par les enseignements théoriques dispensés et les activités professionnelles de terrain. Le ministère de l'Enseignement supérieur et de la Recherche a souhaité définir un cadre général de la formation par les stages en précisant, pour chacun d'eux, les compétences requises et en identifiant de façon détaillée les objectifs transversaux et spécifiques de celles-ci. L'inter-commission a élaboré un ensemble de tableaux de bord spécifiques du type de stage qui permettent le suivi de la progression de l'étudiant dans l'acquisition de compétences professionnelles grâce à une évaluation sommative, formative, formatrice et certificative. Cette évaluation du "savoir-faire" et du "savoir-être" sanctionne l'acquisition de compétences transversales et spécifiques adaptées aux activités propres à chaque parcours professionnel. Pour exemple, dans le cadre de la formation en officine, l'utilisation des tableaux de bord des stages d'application et du stage professionnel (validés par le Collège national des maîtres de stages) atteste de l'intérêt indéniable de ces outils pédagogiques. Deux autres tableaux de bord ont été rédigés, respectivement pour le stage officinal d'initiation et le stage hospitalier (validation CPNES, 2013).

TEG1 Les tableaux de bord sont destinés à être rassemblés pour constituer un carnet de stages (portfolio) dans le but d'observer la progression de l'acquisition des compétences pratiques professionnelles (savoir-faire et savoir-être) de l'étudiant tout au long de sa formation pharmaceutique.

\section{T1 Conclusion}

Le cursus universitaire des étudiants en pharmacie, bien que soumis à une série de réformes, fait preuve d'une grande richesse en matière d'enseignements par rapport aux autres pays européens. Les arrêtés du DFGSP étant sortis en novembre dernier [24], une adaptation verra le jour à la rentrée prochaine. Le président de la République a annoncé la fin du numerus clausus et de la Paces pour la rentrée 2020 [25]. Un nouveau modèle basé sur le système européen LMD est actuellement étudié 
afin de sortir du "gâchis humain" engendré par la Paces. De nombreux acteurs se saisissent de cette problématique et commencent à réfléchir à une nouvelle approche par compétences des enseignements. Nous ne sommes qu'au prémisse de l'amendement du cursus des formations pharmaceutiques.

\section{Notes}

${ }^{1}$ La loi n $84-52$ du 26 janvier 1984 sur l'enseignement supérieur (loi Savary) a défini l'accès à la deuxième année des études de médecine, pharmacie et odontologie en fixant la répartition annuelle entre les établissements du nombre des étudiants admissibles.

${ }^{2}$ Ce programme, qui n'a pas été appliqué, a servi de base au travail de la Commission sur l'intégration des professions médicales et pharmaceutiques au cursus LMD en juin 2006 et au rapport Réflexions et propositions sur la première année des études de médecine, odontologie, de pharmacie et de sage-femme - L1 santé de février 2008, qui a conduit à fixer la répartition et le contenu des enseignements de la première année commune aux études de santé pour les quatre professions : médecine, odontologie, pharmacie et maïeutique.

\section{Références}

[1] Arrêté du 19 juin 1980 régime des études en vue du diplôme d'État de docteur en pharmacie. Journal officiel de la République française du 28 juin 1980.

www.legifrance.gouv.fr/affichTexte.do?cidTexte=JORFTEXT000000674617

[2] Décret $n^{\circ}$ 80-1097 du 24 décembre 1980 fixant les conditions dans lesquelles le diplôme d'État de docteur en pharmacie se substitue au diplôme d'État de pharmacien ainsi que les dispositions transitoires s'appliquant aux étudiants en pharmacie en cours d'études. Journal officiel de la République française du 31 décembre 1980.

www.legifrance.gouv.fr/affichTexte.do?cidTexte=JORFTEXT000000305087\&categorieLien=id

[3] Arrêté du 12 septembre 1985 relatif à l'organisation du régime des études en vue du diplôme d'État de docteur en pharmacie. Journal officiel de la République française du 22 septembre 1985. www.legifrance.gouv.fr/affichTexte.do?cidTexte=JORFTEXT000000678398\&categorieLien=id

[4] Décret $n^{\circ} 73-848$ du 22 août 1973 relatif à l'internat en pharmacie. Journal officiel de la République française du 1er septembre 1973.

www.legifrance.gouv.fr/affichTexte.do?cidTexte=JORFTEXT000000859895\&categorieLien=id

[5] Arrêté du 17 juillet 1987 relatif au régime des études en vue du diplôme d'État de docteur en pharmacie.

www.legifrance.gouv.fr/affichTexte.do?cidTexte=JORFTEXT000000657132\&categorieLien=cid 
[6] Loi n 84-52 du 26 janvier 1984 sur l'enseignement supérieur. Journal officiel de la République française du 27 janvier 1984.

www.legifrance.gouv.fr/affichTexte.do?cidTexte=JORFTEXT000000692733

[7] Comité national d'évaluation. La formation des pharmaciens en France. Juillet 1998. www.cneevaluation.fr/WCNE_pdf/Pharmacie1.pdf

[8] Comité national d'évaluation. La formation des pharmaciens en France. Volume II : les 24 UFR de pharmacie. Décembre 1998. www.cne-evaluation.fr/WCNE_pdf/Pharmacie2.pdf

[9] Arrêté du 14 août 2003 modifiant l'arrêté du 17 juillet 1987 relatif au régime des études en vue du diplôme d'État de docteur en pharmacie. Journal officiel de la République française du 2 octobre 2003. www.legifrance.gouv.fr/affichTexte.do?cidTexte=JORFTEXT000000613031\&dateTexte=

[10] Décret $n^{\circ}$ 2002-482 du 8 avril 2002 portant application au système français d'enseignement supérieur de la construction de l'Espace européen de l'enseignement supérieur. Journal officiel de la République française du 10 avril 2002.

www.legifrance.gouv.fr/affichTexte.do?cidTexte=JORFTEXT000000771048\&categorieLien=id

[11] DGEFP-CPNE. Contrat d'études prospectives dans la pharmacie d'officine. Rapport final. Mars 2006. https://travail-emploi.gouv.fr/IMG/pdf/officine_-_Tome_1.pdf

[12] Ordre national des pharmaciens (ONP). La pharmacie d'officine en France. Bilan et perspectives. Livre blanc. Janvier 2007.

www.ordre.pharmacien.fr/content/download/4906/57542/version/2/file/Livre-blanc-La-pharmacieofficine-en-France-Bilan-et-perspectives.pdf

[13] Commission pédagogique nationale de la première année des études de santé. Rapport. 20 juillet 2003. www.ladocumentationfrancaise.fr/var/storage/rapports-publics/034000580.pdf

[14] Loi n² 2009-833 du 7 juillet 2009 portant création d'une première année commune aux études de santé et facilitant la réorientation des étudiants. Journal officiel de la République française du 8 juillet 2009.

www.legifrance.gouv.fr/affichTexte.do?cidTexte=JORFTEXT000020828203\&categorieLien=id

[15] Arrêté du 28 octobre 2009 relatif à la première année commune aux études de santé. Journal officiel de la République française du 17 novembre 2009.

www.legifrance.gouv.fr/affichTexte.do?cidTexte=JORFTEXT000021276755\&categorieLien=id

[16] Directive 2005/36/CE du Parlement européen et du Conseil du 7 septembre 2005 relative à la reconnaissance des qualifications professionnelles. Journal officiel de l'Union européenne du 30 septembre 2009. https://eur-lex.europa.eu/legalcontent/FR/TXT/PDF/?uri=CELEX:32005L0036\&from=FR

[17] Décret $n^{\circ}$ 2001-37 du 11 janvier 2001 modifiant le décret $n^{\circ} 89-739$ du 12 octobre 1989 relatif aux concours de l'internat de pharmacie. Journal officiel de la République française du 14 janvier 
2001.

www.legifrance.gouv.fr/affichTexte.do?cidTexte=JORFTEXT000000579114\&dateTexte $=20010114$

[18] Arrêté du 23 juin 1998 relatif au Centre national des concours d'internat. Journal officiel de la République française du 20 août 1998.

www.legifrance.gouv.fr/affichTexte.do?cidTexte=JORFTEXT000000207151\&categorieLien=id

[19] Décret n²012-172 du 3 février 2012 relatif au troisième cycle spécialisé des études pharmaceutiques. Journal officiel de la République française du 5 février 2012.

www.legifrance.gouv.fr/affichTexte.do?cidTexte=JORFTEXT000025283453\&categorieLien=id

[20] Décret n² 2017-883 du 9 mai 2017 modifiant les conditions d'exercice et de remplacement au sein des pharmacies à usage intérieur et les modalités d'organisation du développement professionnel continu des professions de santé. Journal officiel de la République française du 10 mai 2017. www.legifrance.gouv.fr/eli/decret/2017/5/9/AFSH1705328D/jo/texte

[21] Décret $n^{\circ}$ 2010-762 du 7 juillet 2010 relatif à la commission pédagogique nationale des études de santé. Journal officiel de la République française du 8 juillet 2010.

www.legifrance.gouv.fr/affichTexte.do?cidTexte=JORFTEXT000022447619\&categorieLien=id

[22] Arrêté du 22 mars 2011 relatif au régime des études en vue du diplôme de formation générale en sciences pharmaceutiques. Journal officiel de la République française du 13 avril 2011.

www.legifrance.gouv.fr/affichTexte.do?cidTexte=JORFTEXT000023850773\&categorieLien=id

[23] Arrêté du 8 avril 2013 relatif au régime des études en vue du diplôme d'État de docteur en pharmacie. Journal officiel de la République française du 26 avril 2013.

www.legifrance.gouv.fr/affichTexte.do?cidTexte=JORFTEXT000027356819\&categorieLien=id

[24] Arrêté du 25 octobre 2018 modifiant l'arrêté du 22 mars 2011 modifié relatif au régime des études en vue du diplôme de formation générale en sciences pharmaceutiques. Version consolidée au 23 novembre 2018. www.legifrance.gouv.fr/affichTexte.do?cidTexte=JORFTEXT000037627425

[25] Tesniere A, Rist S, Riom I. Stratégie de transformation du système de santé. Adapter les formations aux enjeux du système de santé. Rapport final. https://solidarites-

sante.gouv.fr/IMG/pdf/masante2022_rapport_adaptation_des_formations.pdf

Déclaration de liens d'intérêts

Les auteurs déclarent ne pas avoir de liens d'intérêts.

\section{Illustration}

\section{Ade_illus1}

(C) Stefan Schierle/stock.adobe.com 
La première année des études pharmaceutiques était sanctionnée par un concours spécifique, elle a fait l'objet d'une réflexion sur la possibilité de mettre en place un concours commun à d'autres professions de santé.

Sur 1 colonne près de son appel 


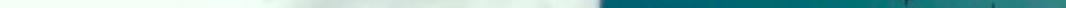

